\title{
One-Year Results of a Treat-and-Extend Regimen of Intravitreal Aflibercept for Polypoidal Choroidal Vasculopathy
}

\author{
Tomoko Tamachi · Takeya Kohno · Manabu Yamamoto • \\ Kumiko Hirayama · Akika Kyo · Nobuhiko Ueda · Michiko Hirabayashi • \\ Kunihiko Shiraki · Shigeru Honda
}

Received: June 5, 2020 / Accepted: October 3, 2020 / Published online: October 15, 2020

(C) The Author(s) 2020

\begin{abstract}
Introduction: To evaluate 1-year outcomes of intravitreal aflibercept (IVA) using a treat-andextend (TAE) regimen for polypoidal choroidal vasculopathy (PCV) and identify the factors for patients whose treatment intervals could be extended.

Methods: Fifty-one eyes of treatment-naïve PCV patients treated with IVA using a TAE regimen for at least 1 year were examined retrospectively. All patients received at least three IVA injections every 5 weeks, and the intervals were then extended by 2 -week adjustments up to 13 weeks. When retinal exudation recurred, the patient was treated with the same regimen, but with a shortened interval of 5 weeks. The main outcome measures were changes in bestcorrected visual acuity (BCVA) and central retinal thickness (CRT) as well as the treatment interval at 1 year.
\end{abstract}

T. Tamachi · T. Kohno (ه) · M. Yamamoto ·

K. Hirayama $\cdot$ A. Kyo $\cdot$ N. Ueda $\cdot$ K. Shiraki .

S. Honda

Department of Ophthalmology and Visual Sciences,

Osaka City University Graduate School of Medicine,

Osaka, Japan

e-mail: takeya@med.osaka-cu.ac.jp

M. Hirabayashi

Department of Ophthalmology, Shiraniwa Hospital, Ikoma, Japan
Results: The mean logarithm of the minimum angle of resolution BCVA improved from $0.24 \pm 0.32$ at baseline to $0.18 \pm 0.31$ at 12 months $(p=0.048)$. The mean CRT decreased from $350.3 \pm 147.7 \mu \mathrm{m}$ at baseline to $215.3 \pm 75.0 \mu \mathrm{m}$ at 4 months $(p<0.001)$, after which it was maintained at this level. At 12 months, the administration interval was 5 weeks in eight eyes (15.7\%), 7 weeks in six eyes $(11.8 \%)$, 9 weeks in two eyes $(3.9 \%)$, 11 weeks in four eyes (7.8\%), and 13 weeks in 31 eyes $(60.8 \%)$. Female sex, a thinner CRT at 6 months, and absence of polypoidal lesions at 12 months were significant factors related to patients whose treatment intervals could be extended without recurrence to 13 weeks.

Conclusion: IVA using a TAE regimen improved visual and anatomical outcomes in eyes with PCV at 1 year using a protocol to adjust the injection intervals specifically for each patient so as to obtain no retinal exudation.

Keywords: Aflibercept; Age-related macular degeneration; Polypoidal choroidal vasculopathy; Treat-and-extend regimen 


\section{Key Summary Points}

PCV treatment remains under discussion.

Based on two large-scale, clinical, controlled studies, the EVEREST II study and the PLANET study, combination therapy with an anti-VEGF drug and PDT and with intravitreal aflibercept (IVA) monotherapy are seen as the initial treatment strategies for treatment-naïve PCV patients.

However, the best treatment regimen that can maintain the improvement achieved during the initial treatment along with a lower clinic and patient burden in routine clinical practice and treatment regimens that are suitable for individualized patients who show a good or a poor response to treatment have yet to be definitively established.

IVA treatments using the TAE regimen resulted in good visual and anatomical outcomes after 1 year of treatment. Overall, $60 \%$ of eyes did not show any recurrence, and in $15 \%$ of eyes, it was not possible to extend the dosing interval beyond 7 weeks. IVA using the TAE regimen may provide suitable injection intervals specifically for each patient so as to obtain no retinal exudation.

Female sex, the presence or absence of exudative changes during the time that the dosing interval was extended to 9 weeks, and the absence of polypoidal lesions on indocyanine green angiography at 12 months may be associated with a good response.

\section{DIGITAL FEATURES}

This article is published with digital features, including a summary slide, to facilitate understanding of the article. To view digital features

for this article go to https://doi.org/10.6084/ m9.figshare.13034252.

\section{INTRODUCTION}

Polypoidal choroidal vasculopathy (PCV) is one of the subtypes of age-related macular degeneration (AMD); it is characterized by a branching vascular network (BVN) and polypoidal lesions (polyps) [1, 2]. This subtype accounts for 23-55\% of Japanese AMD patients [3-5]. During the natural course of PCV with exudation, the number of cases in which the best corrected visual acuity (BCVA) decreases after a year or more increases [6].

PCV treatment includes photodynamic therapy (PDT) and the use of anti-vascular endothelial growth factor (VEGF) drugs such as ranibizumab (Lucentis, Novartis Pharma AG, Basel, Switzerland) and aflibercept (Eylea, Regeneron, Tarrytown, NY, USA, and Bayer HealthCare, Berlin, Germany).

The EVEREST II study compared intravitreal ranibizumab (IVR) monotherapy with combination therapy of IVR and PDT. The combination therapy was superior with regard to BCVA improvement and a smaller number of IVR treatments required after 1 year [7]. In the PLANET study, improvements in visual and functional outcomes were obtained in $>80 \%$ of the patients after 2 years with intravitreal aflibercept (IVA) monotherapy [8].

However, currently, the best treatment regimen to maintain the improvement achieved with initial treatment along with a lower clinic and patient burden in routine clinical practice has not been identified. Similarly, treatment regimens suitable for specific patients have not been yet established.

Various modalities have been used for IVA monotherapy to treat PCV. These include fixed administration at intervals of 2 months after initial administration of three monthly loading treatments [8-11], pro-re-nata (PRN) $[10,12,13]$, and treat-and-extend (TAE) regimens $[14,15]$. Previous studies have reported that fixed administration can result in either under or over treatment in some cases $[9,11]$. 
In contrast, TAE uses a shortened injection interval when exudative changes remain or worsen and an extended injection interval when exudative changes disappear [14-19]. In the current study, the administration interval was extended by 2 weeks when the exudative changes disappeared after at least three monthly loading treatments, with administrations reset to the initial 1-month interval after any recurrence. The aim of this study was to examine both the 1-year results after administering IVA using the TAE regimen and the factors identifying patients whose treatment intervals could be extended to 3 months without any recurrence at 1 year.

\section{METHODS}

The Ethics Committee of the Osaka City University Hospital Institutional Review Board approved this retrospective study (no. 2019-062). All patients provided written, informed consent for the treatment. The study adhered to the tenets of the Declaration of Helsinki and its later amendments or comparable ethical standards.

\section{Study Design}

The medical records of treatment-naïve PCV patients who visited the Department of Ophthalmology, Osaka City University Hospital between January 2013 and August 2014 and were treated by IVA monotherapy were retrospectively reviewed.

\section{Inclusion and Exclusion Criteria}

The subjects were patients aged $\geq 50$ years who had been treated with IVA using the TAE method for at least 1 year. The clinical diagnosis of PCV was based on the indocyanine green angiography (ICGA) finding of polyps.

Polyps were defined as early subretinal hyperfluorescent lesions appearing within 5 min on ICGA associated with BVN and/or a nodular appearance on slit-lamp biomicroscopy. Two retinal specialists (T.K. and M.H.) reviewed the ICGA results independently to determine the presence of polyps and BVN lesions in a blinded manner. If there was disagreement between the readers, a third retinal specialist (K.S.) intervened to decide the result.

Cases with unclear polyps with a history of laser photocoagulation, anti-VEGF drugs, PDT, vitreous surgery, or choroidal neovascularization due to other causes such as high myopia (i.e., refractive error of $\geq-6$ diopters), angioid streaks, inflammation, or trauma were excluded.

\section{Assessment}

At each visit, all patients underwent a comprehensive ophthalmologic examination, which included measurement of BCVA using a Landolt $\mathrm{C}$ acuity chart at $5 \mathrm{~m}$, indirect ophthalmoscopy, slit-lamp biomicroscopy, fundus camera examination (Topcon, Tokyo, Japan), and optical coherence tomography (OCT) (swept-source OCT, DRI OCT-1 Atlantis; Topcon, Tokyo, Japan, or spectral-domain OCT, Spectralis; Heidelberg Engineering, Heidelberg, Germany). Horizontal and vertical scans crossing the fovea and volume scans including the whole area of polyps and BVN were performed. Both standard and enhanced depth imaging protocols were used on Spectralis OCT.

Fluorescein angiography (FA) and ICGA were performed at baseline and at 4 and 12 months using a Heidelberg Retina Angiograph system (Heidelberg Engineering) equipped with a confocal scanning laser ophthalmoscope.

\section{Treatment and Follow-Up Schedule}

IVA administration $(2.0 \mathrm{mg} / 0.05 \mathrm{ml})$ was performed in a sterile environment. All patients received at least three consecutive loading treatments at 5-week intervals. The injections were continued at 5 -week intervals until retinal exudation (i.e., subretinal fluid and/or intraretinal fluid or subretinal hemorrhage) disappeared on OCT or slit-lamp biomicroscopy. The dosing interval was extended up to 13 weeks by increasing the intervals by 2 weeks each time. When retinal exudation 
recurred, the dosing interval was shortened to 5 weeks once again and continued until the retinal exudation disappeared, after which the dosing interval was again increased by 2 weeks each time.

\section{Time Point Setting for Outcome Evaluation}

When using the TAE regimen, the treatment interval was adjusted based on the presence or absence of retinal exudation on OCT and slitlamp biomicroscopy. Thus, the visit times varied depending on the individual patients. In this study, the findings of BCVA, central retinal thickness (CRT), central choroidal thickness (CCT), and presence or absence of retinal exudation were evaluated at baseline, at 4 months (the visit for the first injection after the loading treatments), at 6 months (around 26 weeks, when the injection interval was extended to 9 weeks in patients without recurrence), at 9 months (around 37 weeks, when the injection interval was extended to 11 weeks in patients without recurrence), and at 12 months (visits over 48 weeks).

\section{Outcome Measures}

The BCVA was converted into logMAR units for statistical analysis. The eyes were categorized into three groups on the basis of the BCVA change from baseline (changes of 0.3 or more logMAR units): improved, maintained, and worsened.

The CRT was defined as the distance from the inner limiting membrane (ILM) to the posterior surface of the retinal pigment epithelium (RPE) at the fovea. The CCT was defined as the distance from Bruch's membrane to the border between the suprachoroidal interstitium and the inner side of the sclera at the fovea. The CRT and CCT were measured manually using Heidelberg Eye Explorer software (v.1.0.15.0) or Topcon IMAGEnet 6 software (v. 1.22).

The greatest linear dimension (GLD) was measured as the longest length of the areas that included all polyps and BVNs on ICGA. The GLD, number of polyps, and maximum diameter of polyps were measured manually using Heidelberg Eye Explorer software (v.6.9.5.0). The presence or absence of polyps was evaluated on ICGA at baseline and at 4 and 12 months.

\section{Subgroup Analysis}

The eyes for which the interval of IVA treatment could be extended from 5 to 13 weeks without any recurrence prior to the visit at 12 months were considered good responders. The eyes for which the retinal exudation resolved after at least three monthly initial loading treatments and the treatment interval was extended beyond 7 weeks, but the retinal exudation recurred during follow-up, were considered responders. The eyes in which the retinal exudation remained with monthly IVA, or the retinal exudation resolved, but recurred quickly when the IVA interval was extended to 7 weeks, and thus the interval of IVA could not be extended beyond 7 weeks, were considered poor responders.

The good response group contained the good responders. The recurrence or poor response group contained the responders and poor responders.

The baseline findings of age, sex, GLD, pigment epithelial detachment (PED) of more than one optic disc diameter on fundus camera examination, subretinal hemorrhage (SRH) of more than one optic disc diameter on fundus camera examination, intraretinal fluid on OCT, and number of polyps and maximum diameter of polyps on ICGA were compared between the two groups. LogMAR BCVA, CRT, and CCT at baseline and at 4, 6, 9, and 12 months and the presence or absence of polyps at 4 and 12 months on ICGA were also compared between the two groups.

\section{Statistical Analyses}

The Wilcoxon signed-rank sum test was used to compare the changes in the logMAR BCVA, CRT, and CCT. The parameters were compared between the two groups using the chi-squared test, Fisher's exact test, or Mann-Whitney test, 
Table 1 Patients' characteristics and baseline factors associated with the good response group and the recurrence or poor response group

\begin{tabular}{|c|c|c|c|c|c|}
\hline & Total $(n=51)$ & $\begin{array}{l}\text { Good } \\
\text { response } \\
\text { group } \\
(n=31)\end{array}$ & $\begin{array}{l}\text { Recurrence or } \\
\text { poor } \\
\text { response group } \\
(n=20)\end{array}$ & $\begin{array}{l}\text { Univariate } \\
P \text { value }\end{array}$ & $\begin{array}{l}\text { Multivariate } \\
P \text { value } \\
\text { OD } \\
95 \% \text { CI }\end{array}$ \\
\hline Age (years), mean $\pm S D$ & $74.9 \pm 7.3(52-88)$ & $76 \pm 8.3$ & $73 \pm 5.1$ & 0.017 & $\begin{array}{l}0.077 \\
1.10 \\
0.99-1.22\end{array}$ \\
\hline Sex (female) & $16(31.4 \%)$ & $14(45.3 \%)$ & $2(10.0 \%)$ & 0.008 & $\begin{array}{l}0.007 \\
16.14 \\
2.17-119.91\end{array}$ \\
\hline Left eye/right eye & $21 / 30$ & $11 / 20$ & $10 / 10$ & 0.230 & \\
\hline $\operatorname{GLD}(\mu \mathrm{m})$, mean $\pm S D$ & $\begin{array}{l}4308.5 \pm 2062.2 \\
(523-9753)\end{array}$ & $4142 \pm 1817$ & $4566 \pm 2422$ & 0.802 & \\
\hline $\begin{array}{l}\operatorname{logMAR} B C V A \\
\text { mean } \pm S D\end{array}$ & $0.24 \pm 0.32(-0.18-1.4)$ & $0.32 \pm 0.37$ & $0.13 \pm 0.19$ & 0.159 & $\begin{array}{l}0.063 \\
17.28 \\
0.96-309.96\end{array}$ \\
\hline $\log M A R B C V A \leq 0.15$ (eye) & $26(51.0 \%)$ & $14(45.2 \%)$ & $12(60.0 \%)$ & 0.228 & \\
\hline $\mathrm{CRT}(\mu \mathrm{m})$, mean $\pm \mathrm{SD}$ & $350.3 \pm 147.7(131-862)$ & $353 \pm 157$ & $346 \pm 136$ & 0.969 & \\
\hline $\mathrm{CCT}(\mu \mathrm{m})$, mean $\pm \mathrm{SD}$ & $248.9 \pm 75.8(80-491)$ & $239 \pm 72$ & $264 \pm 80$ & 0.643 & \\
\hline $\begin{array}{l}\text { With PED more than } \\
\text { one optic disc diameter } \\
\text { (eyes) }\end{array}$ & $14(27.4 \%)$ & $5(16.1 \%)$ & $9(45 \%)$ & 0.024 & $\begin{array}{l}0.053 \\
0.20 \\
0.04-1.09\end{array}$ \\
\hline $\begin{array}{l}\text { With SRH more than } \\
\text { one optic disc diameter } \\
\text { (eyes) }\end{array}$ & $10(19.6 \%)$ & $5(16.1 \%)$ & $5(25.0 \%)$ & 0.334 & \\
\hline With intraretinal fluid (eyes) & $18(35.3 \%)$ & $11(35.5 \%)$ & $7(35.0 \%)$ & 0.972 & \\
\hline Polyp number, mean $\pm S D$ & $1.9 \pm 1.4$ & $1.8 \pm 1.2$ & $2.1 \pm 1.7$ & 0.922 & \\
\hline $\begin{array}{l}\text { Polyp diameter }(\mu \mathrm{m}) \\
\text { mean } \pm S D\end{array}$ & $481 \pm 313$ & $472 \pm 359$ & $495 \pm 230$ & 0.429 & \\
\hline
\end{tabular}

$P$ value probability value, $O D$ odds ratio, $C I$ confidence interval, $y$ years, $S D$ standard deviation, $\log M A R \operatorname{logarithm}$ of the minimum angle of resolution, $B C V A$ best-corrected visual acuity, GLD greatest linear dimension, $\mu m$ micrometers, $C R T$ central retinal thickness, $C C T$ central choroidal thickness, GLD greatest linear dimension, PED pigment epithelial detachment, $S R H$ subretinal hemorrhage, Polyp polypoidal lesion

as appropriate. The Bonferroni method was used to correct $P$ values in multiple analyses for changes of BCVA, CRT, CCT, and polyp occlusion. 


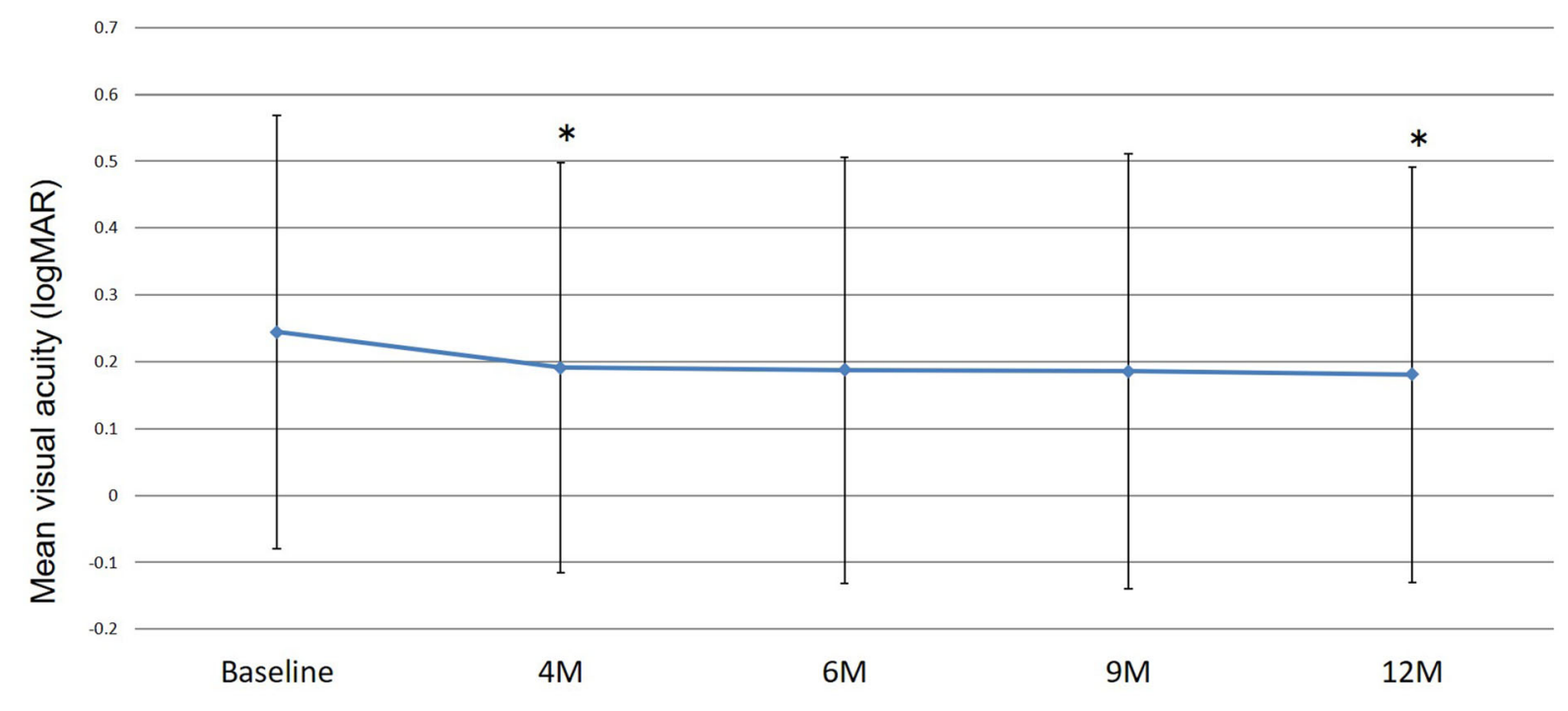

Time after treatment

Fig. 1 Mean $\log M A R$ BCVA over the 1-year course. The mean $\log$ MAR BCVA improves from $0.24 \pm 0.32$ at baseline to $0.19 \pm 0.31$ at 4 months $(p=0.017)$,

To examine the factors at baseline correlated with the TAE response, multivariate logistic regression analysis was performed. Factors with $P<0.2$ on univariate analysis were selected for the multivariate analysis to assess the independent effect of factors on the good response group.

All statistical analyses were performed using SPSS software (IBM, Armonk, NY, USA). The averages of numerical values are presented as means \pm standard deviation (SD) throughout the study. Probability values $<0.05$ were considered significant.

\section{RESULTS}

IVA treatment using the TAE regimen was started in 68 of the 99 eyes in treatment-naïve PCV, with exclusion of 2 eyes due to a large SRH at baseline, 1 eye that was given two monthly administrations, and 28 eyes that were treated using the PRN regimen. After excluding six eyes in which TAE was discontinued because of patients' difficulty attending regular visits or financial reasons, a total of 62 eyes were followed for $>1$ year. FA and ICGA were
$0.19 \pm 0.32$ at 6 months $(p=0.051), 0.19 \pm 0.33$ at 9 months $(p=0.061)$, and $0.18 \pm 0.31$ at 12 months $(p=0.048)\left({ }^{*} p<0.05\right)$

performed in all patients at baseline. Follow-up examinations of FA and ICGA were not performed because of an allergic reaction in three eyes and patients' preference in eight eyes, respectively. Thus, a total of 51 eyes were examined in the current study.

There were no adverse events during the follow-up period. Vitreous hemorrhage was observed in one eye at 11 months in a patient with a poor response.

Patients' characteristics and factors at baseline associated with the good response group and the recurrence or poor response group are shown in Table 1.

Of the 51 eyes evaluated, $31(60.8 \%)$ were in the good response group, and $20(39.2 \%)$ were in the recurrence or poor response group.

\section{Visual Outcome}

The mean BCVA improved from $0.24 \pm 0.32$ at baseline to $0.19 \pm 0.31$ at 4 months $(p=0.017)$, $0.19 \pm 0.32 \quad$ at $\quad 6$ months $\quad(p=0.051)$, $0.19 \pm 0.33$ at 9 months $(p=0.061)$, and $0.18 \pm 0.31$ at 12 months $(p=0.048)$ (Fig. 1$)$. 

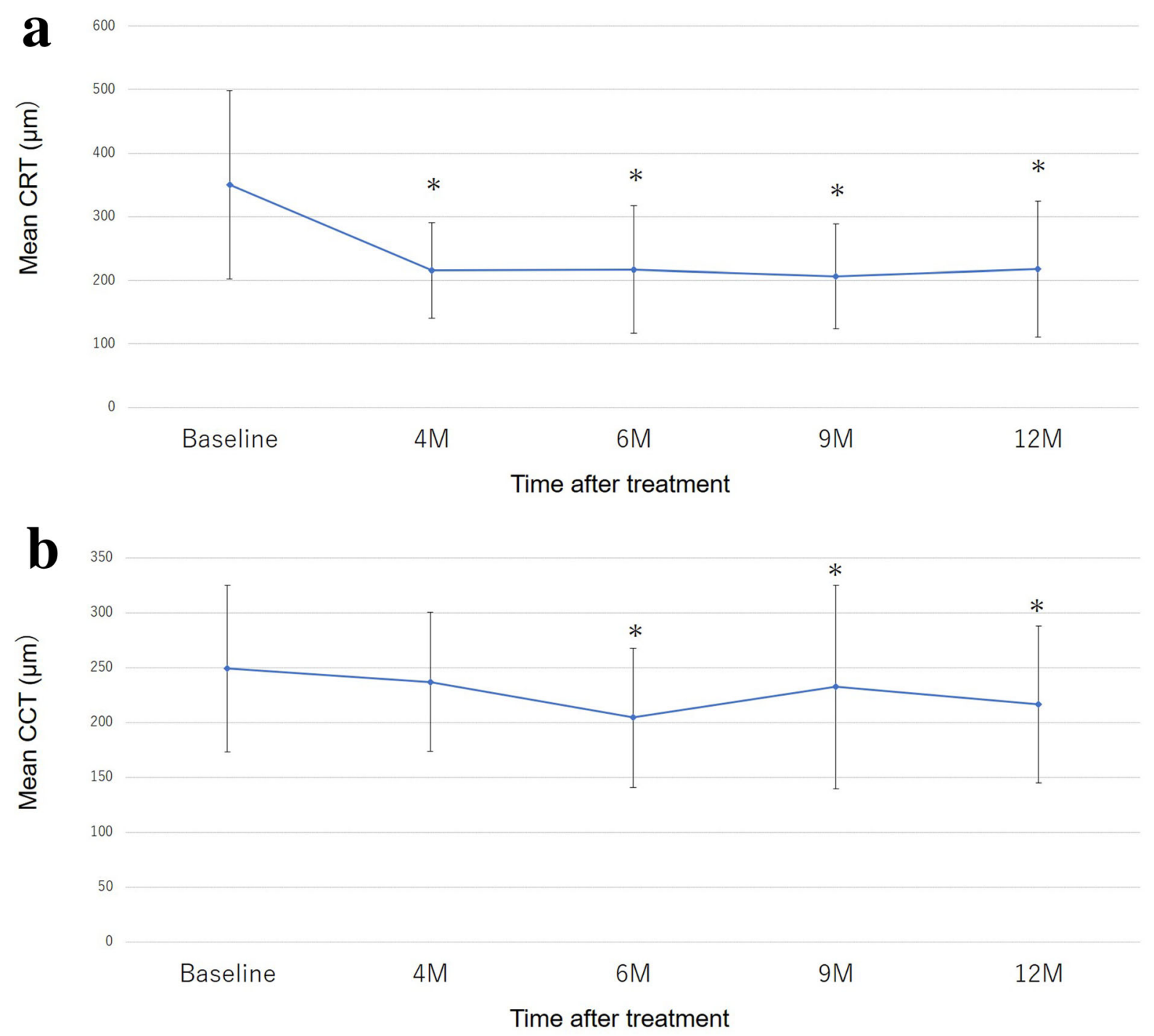

Fig. 2 a Mean CRT over 1 year. The mean CRT is $350.3 \pm 147.7 \mu \mathrm{m}$ at baseline, $215.3 \pm 75.0 \mu \mathrm{m}$ at 4 months $(p<0.001), 217.0 \pm 100.2 \mu \mathrm{m}$ at 6 months $(p<0.001), 206.0 \pm 82.3 \mu \mathrm{m}$ at 9 months $(p<0.001)$, and $217.8 \pm 106.9 \mu \mathrm{m}$ at 12 months $(p<0.001)$ $\left({ }^{*} p<0.001\right)$. b The mean CCT over 1 year. The mean

BCVA improved in seven eyes (13.7\%), whereas it worsened in two eyes (3.9\%) at 12 months.

\section{Anatomical Outcome}

The mean CRT decreased from $350.3 \pm 147.7 \mu \mathrm{m}$ at baseline to $215.3 \pm 75.0 \mu \mathrm{m}$ at 4 months $(p<0.001)$,
CCT is $248.9 \pm 75.8 \mu \mathrm{m}$ at baseline, $236.8 \pm 63.4 \mu \mathrm{m}$ at 4 months $(p=0.231), 204.4 \pm 63.5 \mu \mathrm{m}$ at 6 months $(p<0.001), 232.2 \pm 92.6 \mu \mathrm{m}$ at 9 months $(p=0.039)$, and $216.3 \pm 71.5 \mu \mathrm{m}$ at 12 months $\quad(p<0.001)$ $\left({ }^{*} p<0.05\right)$

which was then maintained at this level (Fig. 2a).

The mean CCT was $248.9 \pm 75.8 \mu \mathrm{m}$ at baseline, $\quad 236.8 \pm 63.4 \mu \mathrm{m} \quad(p=0.231) \quad$ at 4 months, $\quad 204.4 \pm 63.5 \mu \mathrm{m} \quad(p<0.001) \quad$ at 6 months, $\quad 232.2 \pm 92.6 \mu \mathrm{m}$ at 9 months $(p=0.039)$, and $216.3 \pm 71.5 \mu \mathrm{m}(p<0.001)$ at 12 months (Fig. 2b). 
- Retinal exudation at 12 months

- No retinal exudation at 12 months

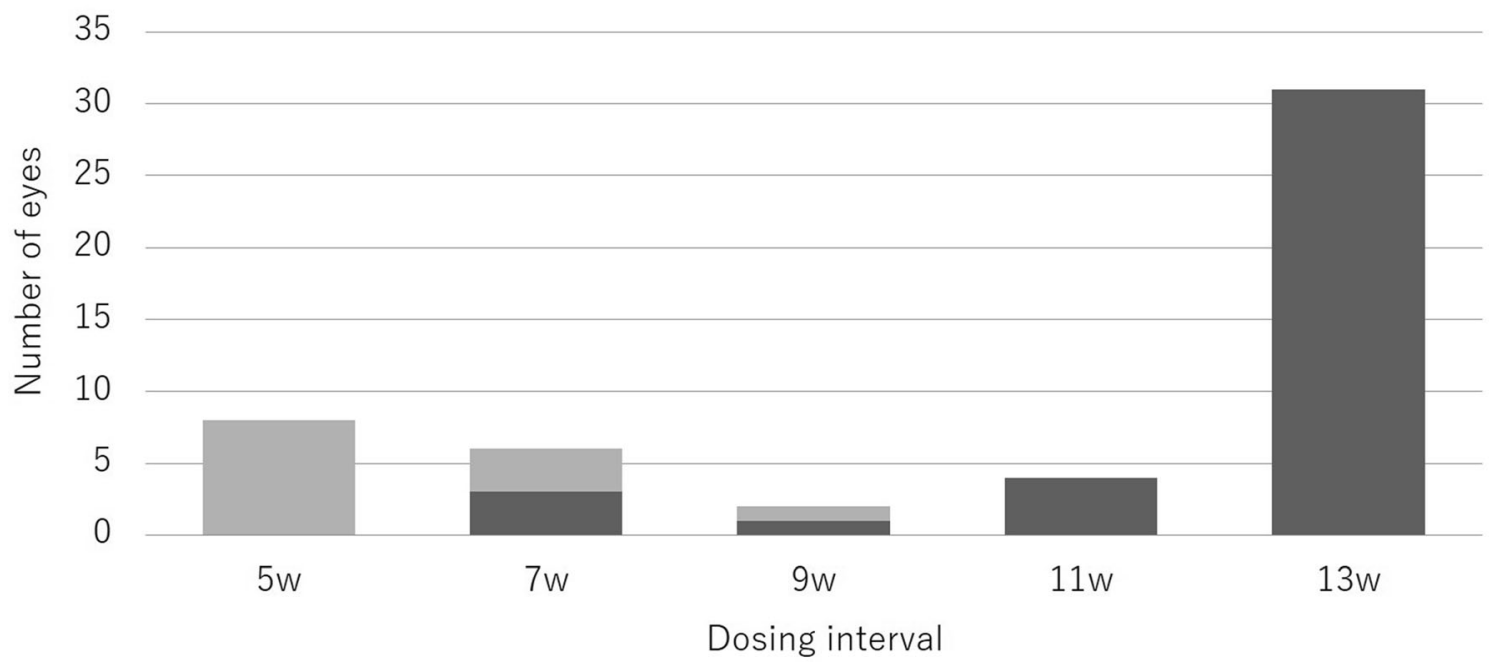

Fig. 3 Dosing interval used prior to 12 months and the presence or absence of exudation at 12 months. The dosing interval at 12 months is 5 weeks in eight eyes (15.7\%), 7 weeks in six eyes (11.8\%), 9 weeks in two eyes $(3.9 \%)$, 11 weeks in four eyes (7.8\%), and 13 weeks in 31 eyes $(60.8 \%)$. The five eyes being treated at 5 -week intervals all have retinal exudation. Of the eyes being treated at 7 -week

Retinal exudation disappeared in 44 eyes $(86.3 \%)$ at 4 months, 38 eyes $(74.5 \%)$ at 6 months, 43 eyes (84.3\%) at 9 months, and 39 eyes $(76.5 \%)$ at 12 months.

\section{Number of Treatments}

The mean number of treatments per 1 year was $7.5 \pm 1.9(6-11)$. A total of 43 eyes $(84.3 \%)$ received three consecutive loading treatments, and then the injection intervals were extended. The other two eyes (3.9\%) received 4 treatments, one eye $(2.0 \%)$ received 5 treatments, one eye $(2.0 \%)$ received 8 treatments, one eye $(2.0 \%)$ received 10 treatments, and three eyes $(5.9 \%)$ received 11 treatments. intervals, three eyes have no retinal exudation, and three eyes have some retinal exudation. Of the two eyes being treated at 9-week intervals, one eye has no retinal exudation, and one eye has retinal exudation. Four eyes being treated at 11 week intervals and 31 eyes being treated at 13 -week intervals have no retinal exudation

\section{Treatment intervals at 12 months and maximum dosing interval during 12 months}

Figure 3 shows the treatment intervals and the presence or absence of exudation at 12 months.

During follow-up, the maximum dosing interval was 5 weeks in 3 eyes (5.9\%), 7 weeks in 5 eyes (9.8\%), 9 weeks in 6 eyes (11.8\%), 11 weeks in 6 eyes $(11.8 \%)$, and 13 weeks in 31 eyes $(60.8 \%)$. Thus, the 8 eyes $(15.7 \%)$ that required maximum dosing of IVA using the TAE regimen at the 5- and 7-week intervals were poor responders, whereas the 31 eyes (60.8\%) being treated with the maximum dosing of IVA using the TAE regimen at 13-week intervals were good responders. The remaining 12 eyes $(23.5 \%)$ with treatment intervals $>7$ weeks with recurrence were responders. 


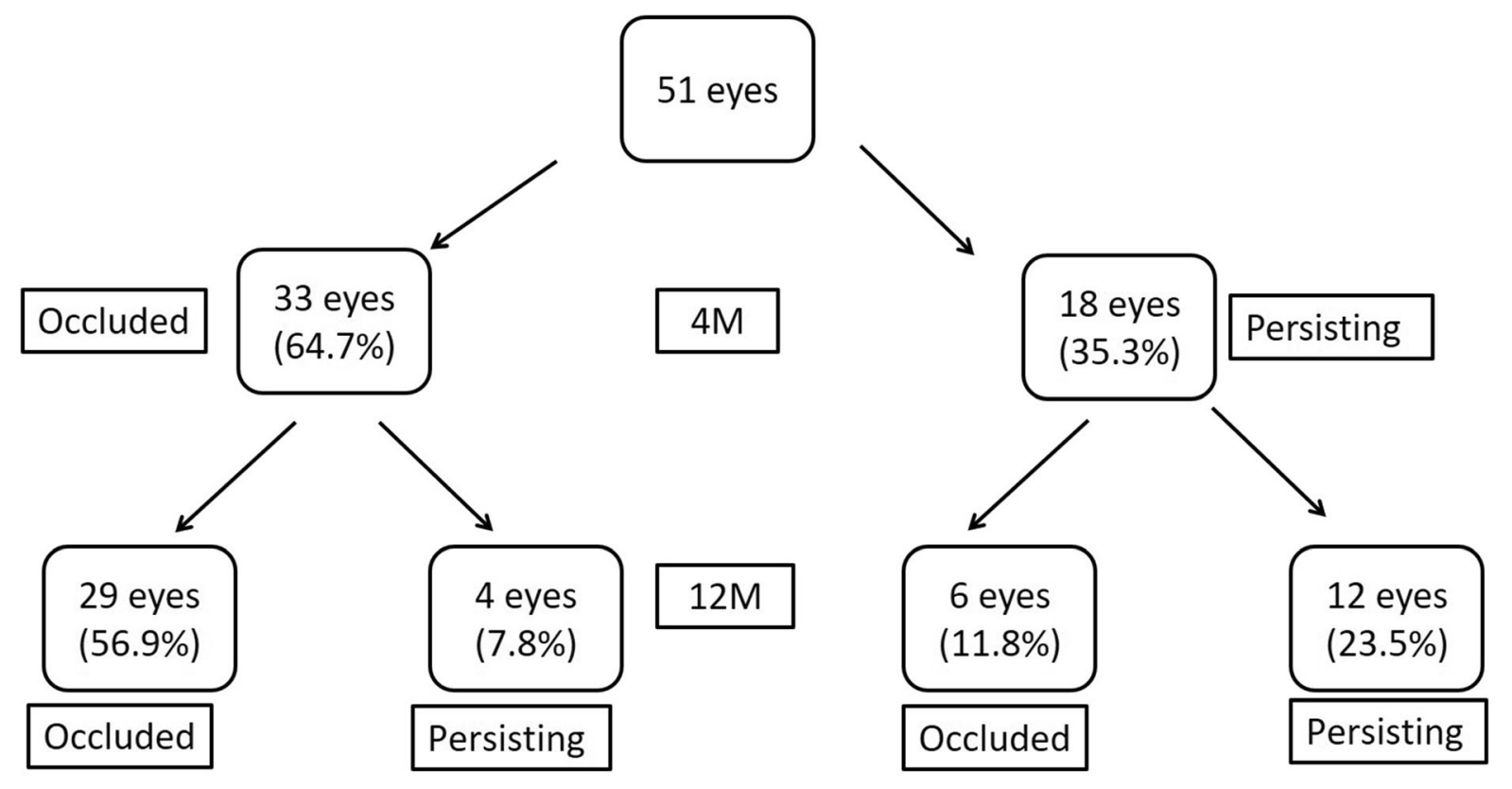

Fig. 4 Changes of polyps. At 4 months, the polyps are occluded in 33 eyes (64.7\%), although 4 of these eyes (7.8\%) subsequently develop polyps at 12 months (1 eye: no retinal exudation; 3 eyes: retinal exudation at 12 months). In the other 18 eyes (35.3\%), the polyps persist at 4 months. These polyps disappear in $6(11.8 \%)$ of the 18 eyes at 12 months (all six eyes: no retinal exudation at 12 months). In the persisting 12 eyes (23.5\%), polyps are

\section{Changes in Polyps on ICGA}

At 4 months, polyps disappeared in 33 eyes (64.7\%). Polyps reappeared in $4(7.8 \%)$ of the 33 eyes at 12 months. On the other hand, polyps persisted in 18 eyes $(35.3 \%)$ at 4 months, with 12 of these eyes $(23.5 \%)$ continuing to have polyps at 12 months. No polyps were seen in 35 eyes (68.6\%) at 12 months (Fig. 4).

\section{Factors Correlated with TAE Treatment}

We examined the relationships of baseline and post-treatment factors with response to IVA treatment. At baseline, univariate analysis showed that there were significant differences in age, sex, and presence or absence of PED. Multivariate logistic regression analysis showed that female sex was a factor for the good response group (Table 1). In the follow-up seen at 12 months (six eyes: no retinal exudation, six eyes: retinal exudation at 12 months). In four of those eyes, the number and/or size of the polyps enlarges from 4 to 12 months (four eyes, retinal exudation). Overall, polyps disappear in 35 eyes (68.6\%) at 12 months. An increase of the polyps from 4 to 12 months is seen in eight eyes (three eyes; responder, five eyes; poor responder)

period, a thinner CRT at 6 months (equivalent to the point of extending the injection interval to 9 weeks in eyes without recurrence) and absent polyps on ICGA at 12 months were significantly correlated with the good response group (Table 2).

\section{DISCUSSION}

This study presents the 1-year results of IVA treatment using the TAE regimen in treatmentnaïve PCV patients. At 12 months, BCVA was either improved or maintained in $96 \%$ of eyes, achieving a retina with no retinal exudation in about $70 \%$ of the eyes. In about $60 \%$ of the eyes, it was possible to extend the treatment intervals to 13 weeks without any recurrence. However, the dosing interval could not be extended beyond 7 weeks in only $15 \%$ of the eyes. 
Table 2 Post-treatment factors associated with the good response group and the recurrence or poor response group

\begin{tabular}{|c|c|c|c|c|}
\hline & $\begin{array}{l}\text { Total } \\
(n=51)\end{array}$ & $\begin{array}{l}\text { Good responder group } \\
(n=31)\end{array}$ & $\begin{array}{l}\text { Recurrence or poor responder group } \\
(n=20)\end{array}$ & $P$ value \\
\hline \multicolumn{5}{|c|}{$\operatorname{logMAR} \mathrm{BCVA}$, mean $\pm \mathrm{SD}$} \\
\hline Baseline & $0.24 \pm 0.32$ & $0.32 \pm 0.37$ & $0.13 \pm 0.19$ & - \\
\hline 4 months & $0.19 \pm 0.31$ & $0.24 \pm 0.36$ & $0.11 \pm 0.19$ & 1.000 \\
\hline 6 months & $0.19 \pm 0.32$ & $0.24 \pm 0.37$ & $0.11 \pm 0.21$ & 1.000 \\
\hline 9 months & $0.19 \pm 0.33$ & $0.22 \pm 0.38$ & $0.13 \pm 0.23$ & 1.000 \\
\hline 12 months & $0.18 \pm 0.31$ & $0.21 \pm 0.36$ & $0.14 \pm 0.21$ & 1.000 \\
\hline \multicolumn{5}{|c|}{$\mathrm{CRT}(\mu \mathrm{m})$, mean $\pm \mathrm{SD}$} \\
\hline Baseline & $350.3 \pm 147.7$ & $353 \pm 157$ & $346 \pm 136$ & - \\
\hline 4 months & $215.3 \pm 75.0$ & $196 \pm 51$ & $245 \pm 96$ & 0.192 \\
\hline 6 months & $217.0 \pm 100.2$ & $185 \pm 59$ & $267 \pm 129$ & 0.020 \\
\hline 9 months & $206.0 \pm 82.3$ & $185 \pm 51$ & $239 \pm 109$ & 0.124 \\
\hline 12 months & $217.8 \pm 106.9$ & $202 \pm 94$ & $242 \pm 123$ & 0.696 \\
\hline \multicolumn{5}{|c|}{$\mathrm{CCT}(\mu \mathrm{m})$, mean $\pm \mathrm{SD}$} \\
\hline Baseline & $248.9 \pm 75.8$ & $239 \pm 72$ & $264 \pm 80$ & - \\
\hline 4 months & $236.8 \pm 63.4$ & $236 \pm 62$ & $238 \pm 67$ & 1.000 \\
\hline 6 months & $204.4 \pm 63.5$ & $194 \pm 68$ & $221 \pm 54$ & 0.812 \\
\hline 9 months & $232.2 \pm 92.6$ & $224 \pm 90$ & $245 \pm 98$ & 1.000 \\
\hline 12 months & $216.3 \pm 71.5$ & $207 \pm 76$ & $230 \pm 64$ & 1.000 \\
\hline \multicolumn{5}{|c|}{ Polyp occlusion (eyes) } \\
\hline 4 months & & $23(74.2 \%)$ & $10(50.0 \%)$ & 0.156 \\
\hline 12 months & & 27 (87.1\%) & $8(40.0 \%)$ & $<0.001$ \\
\hline
\end{tabular}

$P$ value probability value, $S D$ standard deviation, $\log M A R$ logarithm of the minimum angle of resolution, $B C V A$ bestcorrected visual acuity, $G L D$ greatest linear dimension, $\mu m$ micrometer, $C R T$ central retinal thickness, $C C T$ central choroidal thickness, GLD greatest linear dimension, PED pigment epithelial detachment, Polyp polypoidal lesion

A previous comparison of the effects of ranibizumab and aflibercept monotherapy on treatment-naïve PCV showed that, although the BCVA improved in both groups over the course of 1 year, the occlusion rate for the polyps was $21.6 \%$ in the IVR and $39.5 \%$ in the IVA groups. Aflibercept monotherapy is considered to be one of the better options for PCV treatment [20]. The standard IVA treatment regimen uses fixed intervals of every 2 months after the initial three monthly loading injections [21]. When using this standard regimen, the 1-year results indicated that BCVA improved and was maintained in $95 \%$ or more. In addition, there was complete occlusion of the polyps in $40-70 \%$, ultimately achieving a retina with no retinal exudation in $60-80 \%$ of the patients. The average number of treatments has been reported to be 7.1-8.1 [8-11]. Hosokawa et al. studied the TAE regimen and found that BCVA improved 
and was maintained in $97.3 \%$, with complete occlusion of the polyps occurring in $51.4 \%$ of the patients. Subsequently, a retina with no retinal exudation was achieved in $73.0 \%$, with an average of 8.2 treatments [14]. In the present study, the BCVA improved and was maintained in $96 \%$, with complete occlusion of polyps in $68.6 \%$, and a retina with no retinal was achieved in $76.5 \%$, with an average of 7.5 treatments. There were 26 eyes $(51.0 \%)$ with a logMAR BCVA of 0.15 or less (decimal BCVA of 0.7 or more) in the current study. In the majority of previous prospective studies, patients with a pretreatment decimal BCVA $<$ 0.5 were included [21], and there were only a few studies that treated patients with AMD including PCV that showed good BCVA $[22,23]$. Thus, the results of the current study may provide useful information with regard to IVA treatments of PCV patients in clinical practice.

In the ALTAIR study, the administration of IVA using the TAE regimen at 2- and 4-week intervals was compared in Japanese patients with exudative AMD. The study results showed that the administration interval was extended to 3 months in about $60 \%$ of cases during both the 1 st and 2 nd years, which led to a reduction in the clinic and patient burden, along with visual anatomical outcomes that were maintained after the initial IVA doses [17]. In the PLANET study protocol, which was designed to study the treatment of $\mathrm{PCV}$, the guidelines permitted physicians to extend the injection intervals to $>10$ weeks during the second year. As a result, this interval was extended in $>50 \%$ of eyes [8]. Hosokawa et al. reported that, when using the TAE regimen, the dosing interval could be extended to 12 weeks in $59.5 \%$ of eyes during the course of 1 year [14]. In the current study, the injection interval was also extended to 13 weeks in $60.8 \%$ of eyes treated for up to 1 year.

In the current study, the shortest dosing period was set to 5 weeks, with the dosing interval extended every 2 weeks or reset to 5 weeks on recurrence. This is different from other studies in which the shortest injection period was set to 4 weeks. Since the Japanese insurance system requires that the shortest dosing interval of anti-VEGF must be 4 weeks, we only had a margin of 1 week prior to the shortest interval (5 weeks). Due to patients' preferences, this often made it difficult to schedule visits at 5 weeks, especially when there was worsening of the exudative changes. In the current study, a retina with no retinal exudation was achieved in 43 eyes (84.3\%) after the loading treatment. The shortest interval, which was based on visits at 5 weeks, did not significantly affect the results compared to those previously reported for the 4-week intervals $[14,24]$.

It has been reported that several factors may affect the duration and number of ant-VEGF injections for AMD. In the Harbor study, a post hoc analysis showed that the state of the retina, with or without retinal exudation, after the initial two injections appears to be a factor related to the number of IVR injections [25]. Morizane-Hosokawa et al. reported that the presence or absence of polyps after the loading treatment might be related to the recurrence of the exudative changes that occur during IVA treatment when using the TAE regimen [24].

In the present study, the comparison of the good response group and the recurrence or poor response group showed that female sex was an independent factor related to good responders at baseline (Table 1). Of the post-treatment factors, a thinner CRT at 6 months and absent polyps on ICGA at 12 months were significantly related to the good response group. At 6 months, 38 eyes had no retinal exudation. In $31(81.6 \%)$ of these 38 eyes, it was possible to extend the injection intervals up to 13 weeks, with a retina with no retinal exudation subsequently achieved in $33(86.8 \%)$ of the 38 eyes at 12 months. Thus, the presence or absence of exudative changes during the time that the dosing interval was extended to 9 weeks was a factor associated with good responders. These findings should help patients become aware of future treatment prospects, and, in addition, physicians will be able to determine the clinical settings for the long-term treatment plans.

The results for the administration of IVA using the TAE regimen highlight the ability to differentiate poor responders from good responders and responders. The dosing intervals 
could not be extended to $>7$ weeks in eight eyes (15.7\%) during 12 months.

In the PLANET study, the number of patients who required rescue PDT because of being refractory to IVA and the continuous use of two monthly IVA treatments increased to $6 \%$ of patients at 3 months, $13 \%$ at 1 year, and $16 \%$ at 2 years.

Although rescue PDT was performed in eyes with polyps being given IVA, this study failed to clarify the clinical significance of the rescue PDT compared with monthly IVA during the 2 years of follow-up [8]. On the other hand, it has been reported that PDT was effective in achieving a retina with no retinal exudation in cases that showed a poor response to IVR monotherapy [26]. In the EVEREST II study, approximately three or more administrations of PDT were required during a year in about $12 \%$ of cases, with these patients showing resistance to the combined therapy of anti-VEGF drugs and PDT [7]. In the present study, although monthly IVA was performed, polyps developed in five of the eight eyes after 4-12 months of treatment. Thus, the treatment strategies for poor responders when using anti-VEGF need to be considered differently from those that have a good response to these treatments. Further studies will need to be undertaken to clarify the clinical features of these poor responders and possible associated genetic factors. These studies will also need to reconsider the retreatment criteria based on the association between the presence or absence of retinal exudation and other clinical biomarkers, such as the enlargement of PED, presence of fluid under the RPE, and/or development and changes in the blood flows as seen on OCT angiography. Moreover, these additional studies will need to clarify what an adequate regimen consists of in these types of poor responders, such as the timing of PDT, and the use of anti-VEGF treatments after PDT, including the use of continuous treatments.

The limitations of this study include the retrospective nature, the small cohort, and the lack of a control group. In addition, since the follow-up was for only 1 year, long-term evaluation will be needed.

\section{CONCLUSIONS}

IVA treatments using the TAE regimen in untreated PCV resulted in good visual and anatomical outcomes after 1 year of treatment. Overall, 60\% of eyes did not exhibit any recurrence, with eight eyes (15.7\%) unable to extend the dosing interval beyond 7 weeks. Administration of IVA using the TAE regimen may provide suitable injection intervals specifically for each patient so as to obtain no retinal exudation. Female sex, the presence or absence of exudation with injection intervals of 2 months, and absence of polyps at 12 months on ICGA were potentially associated with whether the dosing interval could be extended up to 3 months without recurrence during 1 year.

\section{ACKNOWLEDGEMENTS}

We thank the participants of the study.

Funding No funding or sponsorship was received for this study or publication of this article.

Medical Writing, Editorial, and Other Assistance The authors thank Forte Science Communications (Tokyo, Japan) for editorial support.

Authorship All named authors meet the International Committee of Medical Journal Editors (ICMJE) criteria for authorship for this article, take responsibility for the integrity of the work as a whole, and have given their approval for this version to be published.

Prior Presentation Presented in part at: Association for Research in Vision and Ophthalmology Annual Meeting, May 2017, Baltimore, MD, USA.

Disclosures Dr. T. Kohno reports lecture fees or honoraria, travel fees or reimbursements from Novartis, Bayer, Santen, and Senjyu outside of the submitted work. Dr. M. Yamamoto reports lecture fees or honoraria, travel fees or reimbursements from Novartis, Bayer, Santen, 
Alcon, and Senjyu outside of the submitted work. Dr. S. Honda reports lecture fees or honoraria, travel fees or reimbursements from Novartis, Bayer, Santen, Alcon, and Senjyu outside of the submitted work. Tomoko Tamachi, Kumiko Hirayama, Akika Kyo, Nobuhiko Ueda, Michiko Hirabayashi and Kunihiko Shiraki declare no conflict of interest.

Compliance with Ethics Guidelines The Ethics Committee of the Osaka City University Hospital Institutional Review Board approved this study. (No. 2019-s062) All patients provided written, informed consent for the treatment. The study adhered to the 1964 Helsinki Declaration and its later amendments or comparable ethical standards.

Data Availability All authors had full access to all of the data in this study and take complete responsibility for the integrity of the data and accuracy of the data analysis. The datasets generated and/or analyzed during the current study are available from the corresponding author on reasonable request.

Open Access. This article is licensed under a Creative Commons Attribution-NonCommercial 4.0 International License, which permits any non-commercial use, sharing, adaptation, distribution and reproduction in any medium or format, as long as you give appropriate credit to the original author(s) and the source, provide a link to the Creative Commons licence, and indicate if changes were made. The images or other third party material in this article are included in the article's Creative Commons licence, unless indicated otherwise in a credit line to the material. If material is not included in the article's Creative Commons licence and your intended use is not permitted by statutory regulation or exceeds the permitted use, you will need to obtain permission directly from the copyright holder. To view a copy of this licence, visit http://creativecommons.org/licenses/by$\mathrm{nc} / 4.0 /$.

\section{REFERENCES}

1. Yannuzzi LA, Ciardella A, Spaide RF, et al. The expanding clinical spectrum of idiopathic polypoidal choroidal vasculopathy. Arch Ophthalmol. 1997;115:478-85.

2. Spaide RF, Yannuzzi LA, Slakter JS, et al. Indocyanine green videoangiography of idiopathic polypoidal choroidal vasculopathy. Retina. 1995;15: 100-10.

3. Sho K, Takahashi K, Yamada H, et al. Polypoidal choroidal vasculopathy: incidence, demographic features, and clinical characteristics. Arch Ophthalmol. 2003;121:1392-6.

4. Obata R, Yanagi Y, Kami J, et al. Polypoidal choroidal vasculopathy and retinochoroidal anastomosis in Japanese patients eligible for photodynamic therapy for exudative age-related macular degeneration. Jpn J Ophthalmol. 2006;50:354-60.

5. Maruko I, Iida T, Saito M, et al. Clinical characteristics of exudative age-related macular degeneration in Japanese patients. Am J Ophthalmol. 2015;144: 15-22.

6. Cheung CM, Yang E, Lee WK, et al. The natural history of polypoidal choroidal vasculopathy: a multi-center series of untreated Asian patients. Graefes Arch Clin Exp Ophthalmol. 2015;253: 2075-85.

7. Koh A, Lai TYY, Takahashi K, et al. Efficacy and safety of ranibizumab with or without verteporfin photodynamic therapy for polypoidal choroidal vasculopathy: a randomized clinical trial. JAMA Ophthalmol. 2017;135:1206-13.

8. Yamamoto A, Okada AA, Kano M, et al. One-year results of intravitreal aflibercept for polypoidal choroidal vasculopathy. Ophthalmology. 2015;122: 1866-72.

9. Hara C, Sawa M, Sayanagi K, et al. One-year results of intravitreal aflibercept for polypoidal choroidal vasculopathy. Retina. 2016;36:37-45.

10. Inoue M, Yamane S, Taoka R, et al. Aflibercept for polypoidal choroidal vasculopathy: as needed versus fixed interval dosing. Retina. 2018;36:1527-34.

11. Lee WK, Iida T, Ogura Y, et al. Efficacy and safety of intravitreal aflibercept for polypoidal choroidal vasculopathy in the PLANET study: a randomized clinical trial. JAMA Ophthalmol. 2018;136:786-93.

12. Lee JE, Shin JP, Kim HW, et al. Efficacy of fixeddosing aflibercept for treating polypoidal choroidal vasculopathy: 1-year results of the VAULT study. 
Graefes Arch Clin Exp Ophthalmol. 2017;255: 493-502.

13. Maruyama-Inoue $M$, Sato $S$, Yamane $S$, et al. Intravitreal injection of aflibercept inpatients with polypoidal choroidal vasculopathy: a 3-year followup. Retina. 2018;38:2001-9.

14. Hosokawa M, Morizane Y, Hirano M, et al. One-year outcomes of a treat-and-extend regimen of intravitreal aflibercept for polypoidal choroidal vasculopathy. Jpn J Ophthalmol. 2017;61:150-8.

15. Morimoto M, Matsumoto H, Mimura K, et al. Twoyear results of a treat-and-extend regimen with aflibercept for polypoidal choroidal vasculopathy. Graefes Arch Clin Exp Ophthalmol. 2017;255: 1891-7.

16. Yamamoto A, Okada AA, Nakayama M, et al. Oneyear outcomes of a treat-and-extend regimen of aflibercept for exudative age-related macular degeneration. Ophthalmologica. 2017;237:139-44.

17. Ohji M, Kanji T, Okada AA, et al. Efficacy and safety of intravitreal aflibercept treat-and-extend regimens in exudative age-related macular degeneration: 52and 96-week findings from ALTAIR: a randomized controlled trial. Adv Ther. 2020;37:1173-8. https:// doi.org/10.1007/s12325-020-01236-X.

18. Ohnaka M, Nagai $Y$, Sho K, et al. A modified treatand-extend regimen of aflibercept for treatmentnaïve patients with neovascular age-related macular degeneration. Graefes Arch Clin Exp Ophthalmol. 2017;255:657-64.

19. Maruko I, Ogasawara M, Yamamoto A, et al. Twoyear outcomes of treat-and-extend intravitreal aflibercept for exudative age-related macular degeneration: a prospective study. Ophthalmol Retina. 2020;4:767-76.

20. Cho HJ, Kim KM, Kim HS, et al. Intravitreal aflibercept and ranibizumab injections for polypoidal choroidal vasculopathy. Am J Ophthalmol. 2016;165:1-6.

21. Schmidt-Erfurth U, Kaiser PK, Korobelnik JF, et al. Intravitreal aflibercept injection for neovascular age-related macular degeneration: ninety-six-week results of the VIEW studies. Ophthalmology. 2014;121:193-201.

22. Mori R, Tanaka K, Yuzawa M. Factors predicting 2-year treatment results of ranibizumab therapy for polypoidal choroidal vasculopathy in eyes with good baseline visual acuity. Medicine (Baltimore). 2018;9:e11188.

23. Kato A, Yasukawa $\mathrm{T}$, Suga $\mathrm{K}$, et al. Intravitreal ranibizumab for patients with neovascular age-related macular degeneration with good baseline visual acuity. Ophthalmologica. 2015;233:27-34.

24. Morizane-Hosokawa M, Morizane Y, Kimura S, et al. Impact of polyp regression on 2-year outcomes of intravitreal aflibercept injections: a treat-and-extend regimen for polypoidal choroidal vasculopathy. Acta Med Okayama. 2018;72:379-85.

25. Bogunovic H, Waldstein SM, Schlegl T, et al. Prediction of anti-VEGF treatment requirements in neovascular AMD using a machine learning approach. Invest Ophthalmol Vis Sci. 2017;58: 3240-8.

26. Cho M, Barbazetto IA, Freund KB. Refractory neovascular age-related macular degeneration secondary to polypoidal choroidal vasculopathy. Am J Ophthalmol. 2009;148:70-8. 